J. Math. Soc. Japan

Vol. 20, Nos. 1-2, 1968

\title{
The congruence monodromy problems
}

\author{
Dedicated to Professor Shôkichi Iyanaga on his 60 th birthday
}

By Yasutaka IHARA

(Received Aug. 31, 1967)

\section{Introduction}

This is a summary of the forthcoming lecture note [1]. All details and proofs of the theorems will be given in [1], and are omitted here.

$\S 0$-1. The problems. Let $G=\operatorname{PSL}_{2}(R) \times \operatorname{PSL}_{2}\left(k_{\mathfrak{p}}\right)$, where $R$ and $k_{\mathfrak{p}}$ are the real number field and a $\mathfrak{p}$-adic number field with $N \mathfrak{p}=q$ respectively, and $\mathrm{PSL}_{2}=\mathrm{SL}_{2} / \pm 1$. Let $\Gamma$ be a torsion-free discrete subgroup of $G$ with compact quotient, having a dense image of projection in each component of $G$. Our subject is such a discrete subgroup $\Gamma$. This study was motivated by the following series of conjectures which were suggested by our previous work [2]*. Since our group $\Gamma$ is essentially nonabelian (see $\S 1-5$, property (iv)), the readers will see that, by our conjectures, $\Gamma$ would describe a "non-abelian class field theory" over an algebraic function field of one variable with finite constant field $F_{q^{2}}$. We would like to call the problems of determination of the validity of these conjectures, the congruence monodromy problems.

Conjectures**. With each $\Gamma$, we can associate an algebraic function field $K$ of one variable with finite constant field $F_{q^{2}}$ and with genus $g \geqq 2$, and a finite set $\mathfrak{S}(K)$ consisting of $(q-1)(g-1)$ prime divisors of $K$ of degree one over $F_{q^{2}}$, satisfying the following properties. Here, the elements of $\subseteq(K)$ are called the exceptional prime divisors, while all other prime divisors of $K$ are called the ordinary prime divisors.

CONJECTURE 1. The ordinary prime divisors $P$ of $K$ are in one-to-one correspondence with the pairs $\left\{\gamma_{P}^{ \pm 1}\right\}_{\Gamma}$ of mutually inverse primitive elliptic conjugacy classes of $\Gamma$ (see $\S 1$ for the definitions).

CONJECTURE 2. The finite unramified extensions $K^{\prime}$ of $K$, in which all $(q-1)(g-1)$ exceptional prime divisors of $K$ are decomposed completely, are in

* The proofs of results stated in [2] will also be given in [1]. There is some overlap between a part of $\S 2$ of [2] and $\S 1$ of this paper.

** See also $\S 3$. 
one-to-one correspondence with the subgroups $\Gamma^{\prime}$ of $\Gamma$ with finite indices. Moreover, this one-to-one correspondence satisfies the Galois theory.

CONJECTURE 3. The law of decomposition of ordinary prime divisors $P$ of $K$ in $K^{\prime}$ is described by the corresponding $\left\{\gamma_{P}^{ \pm 1}\right\}_{\Gamma}$ and $\Gamma^{\prime}$. Namely, decompose the $\Gamma$-conjugacy class $\left\{\gamma_{P}\right\}_{\Gamma}$ into a disjoint union of $\Gamma^{\prime}$-conjugacy classes:

$$
\left\{\gamma_{P}\right\}_{\Gamma}=\left\{\gamma_{P, 1}\right\}_{\Gamma^{\prime}} \cup \cdots \cup\left\{\gamma_{P, t}\right\}_{\Gamma^{\prime}},
$$

and for each $i$, let $f_{i}$ be the smallest positive integer such that $\gamma_{P, i}^{f i}$ is contained in $\Gamma^{\prime}$. Then we have $\sum_{i=1}^{t} f_{i}=\left(\Gamma: \Gamma^{\prime}\right)$, and our conjecture asserts that the decomposition of $P$ in $K^{\prime}$ is of type:

$$
P=P_{1}^{\prime} P_{2}^{\prime} \cdots P_{t}^{\prime},
$$

where $P_{i}^{\prime}(1 \leqq i \leqq t)$ are prime divisors of $K^{\prime}$ with relative degrees $f_{i}(1 \leqq i \leqq t)$ respectively.

$\S 0-2$. Results. Now, our results, summarized in $\S 1$ and $\S 2$, are still far from the solution of the problems, but seem encouraging.

In $\S 1$, we shall define primitive elliptic conjugacy classes $P$ of $\Gamma$, their degrees $\operatorname{deg} P$, and the $\zeta$ function $\zeta_{\Gamma}(u)=\prod_{P}\left(1-u^{\operatorname{deg} P}\right)^{-1}$, where $P$ runs over the pairs of mutually inverse primitive elliptic conjugacy classes. Then, the result of the computation of $\zeta_{\Gamma}(u)$ is given in Theorem 1, which states that $\zeta_{\Gamma}(u)$ is a rational function of $u$ of the form:

$$
\zeta_{\Gamma}(u)=\frac{\prod_{i=1}^{g}\left(1-\alpha_{i} u\right)\left(1-\alpha_{\imath}^{\prime} u\right)}{(1-u)\left(1-q^{2} u\right)} \times(1-u)^{(q-1)(g-1)},
$$

where $\alpha_{i} \alpha_{i}^{\prime}=q^{2}$ for $1 \leqq i \leqq g$, and $g(\geqq 2)$ is the genus of a certain algebraic function field $L_{\theta}$ of one variable over the complex number field $C$. This agrees with conjecture 1 , because if conjecture 1 is true, then $\zeta_{\Gamma}(u)$ must be of this form, with the first factor equal to the congruence $\zeta$ function of $K$ over $F_{q^{2}}$.

Now, we expect that the questioned field $K$ is obtained by some "reduction $\bmod \Re$ " of the function field $L_{\theta}$ (the definition of $L_{0}$ is given in $\S 2-1$ ), and so, our first problem is to lower the field of constants of $L_{0}$. However, we find that it is more essential to consider some infinite extension $L$ of $L_{\theta}$ on which the group $G_{\mathfrak{p}}=\mathrm{PSL}_{2}\left(k_{\mathfrak{p}}\right)$ acts as an automorphism group over $C$, and to lower the field of constants of $L$. Thus, in $\S 2-1$, we shall define what we call $a G_{p}$-field; Roughly speaking, it is a field, one dimensional and infinitely generated over its constant field, on which the group $G_{\mathfrak{p}}=\operatorname{PSL}_{2}\left(k_{\mathfrak{p}}\right)$ acts as an automorphism group. Then, we shall show that $G_{p}$-fields over the complex number field $C$ are in one-to-one correspondence with the group $\Gamma$ (Theorem 2). 
Now, our main result is that a $G_{\mathfrak{p}}$-field over $C$ is always the constant field extension of some $G_{\mathfrak{p}}$-field over an algebraic number field of finite degree, and that if the former is irreducible (see $\$ 2-2$ ), then the latter is essentially unique (Theorem 4). Thus, it seems that our problems are reduced to some "arith metic theory" of $G_{p}$-fields over algebraic number fields.

In $\S 3$, our conjectures are repeated a little more precisely, and in $\S 4-1$, some examples of $\Gamma$, which are obtained from certain quaternion algebra, are given. For these $\Gamma$, the fields $L_{0}$ belong to those classes of function fields. which have been studied in detail by G. Shimura [3], but even in these cases neither of our conjectures have been proved*. The only example for which our conjectures are partly proved is given in $\S 4-2$.

The author would like to express his hearty thanks particularly to Professor G. Shimura, and to Professors A. Weil and R.P. Langlands, for their interest in this subject and valuable discussions with the author during his stay in Princeton in 1965-1967.

Throughout the following, for any element $\gamma$ of any group $\Gamma,\{\gamma\}_{\Gamma}$ will denote the conjugacy class of $\Gamma$ containing $\gamma$.

\section{$\S 1$. Group $\Gamma$ and its $\zeta$ function}

$\S 1$ 1. Let $R$ be the field of real numbers, and put $G_{R}=\mathrm{PSL}_{2}(R)=\mathrm{SL}_{2}(R) / \pm 1$. Let, on the other hand, $k_{\mathfrak{p}}$ be a $\mathfrak{p}$-adic number field with the ring of integers $\mathfrak{p}_{\mathfrak{p}}$ and the maximal ideal $\mathfrak{p}$ of $\mathfrak{p}_{\mathfrak{p}}$ with $N \mathfrak{p}=q$, and put $G_{\mathfrak{p}}=\operatorname{PSL}_{2}\left(k_{\mathfrak{p}}\right)=\operatorname{SL}_{2}\left(k_{\mathfrak{p}}\right)_{/}^{\prime} \pm 1$. We form the direct product $G=G_{R} \times G_{p}$, and consider it as a topological group in a natural manner. For any subset $S$ of $G$, we denote by $S_{R}$ resp. $S_{p}$ the images of the set-theoretical projections of $S$ to $G_{R}$ resp. $G_{\mathfrak{p}}$. Thus in particular, for any element $x$ of $G, x_{R}$ resp. $x_{p}$ are the real resp. p-adic components of $x ; x=x_{R} \times x_{p}$.

Now, let $\Gamma$ be a subgroup of $G$ satisfying the following conditions.

( $\Gamma 1)$ The projection maps $\Gamma \rightarrow \Gamma_{R}, \Gamma \rightarrow \Gamma_{p}$ are injective, and the images: $\Gamma_{R}, \Gamma_{\mathfrak{p}}$ are dense in $G_{R}, G_{\mathfrak{p}}$ respectively.

( $\Gamma 2) \quad \Gamma$ is discrete in $G$, and the quotient $G / \Gamma$ is compact.

Actually, these conditions are not independent; in fact by the simplicity of the groups $G_{R}, G_{\mathfrak{p}}$, we can show easily that the injectivities of the projection maps $\Gamma \rightarrow \Gamma_{R}, \Gamma \rightarrow \Gamma_{\mathfrak{p}}$ are the consequences of the rest of the conditions in $(\Gamma 1)$, and $(\Gamma 2)$. By this remark, it becomes clear that if $\Gamma$ is a subgroup of $G$ satisfying $(\Gamma 1)$ and $(\Gamma 2)$, then subgroups $\Gamma^{\prime}$ of $G$ which are commensurable with.

* But we get some useful informations from his results [3], which are very encouraging. See $\S 4-1$. 
$\Gamma$ also satisfy $(\Gamma 1)$ and $(\Gamma 2)$.

Put

$$
\begin{aligned}
& U_{\mathfrak{p}}=\mathrm{PSL}_{2}\left(\mathfrak{o}_{\mathfrak{p}}\right)=\mathrm{SL}_{2}\left(\mathfrak{o}_{\mathfrak{p}}\right) / \pm 1, \\
& \Gamma^{0}=\Gamma \cap\left(G_{R} \times U_{\mathfrak{p}}\right),
\end{aligned}
$$

and let $\Gamma_{R}^{0}$ be the projection of $\Gamma^{0}$ to $G_{R}$.

Then, under $(\Gamma 1)$, the condition $(\Gamma 2)$ is equivalent to the following:

$\left(\Gamma 2^{\prime}\right) \quad \Gamma_{R}^{0}$ is discrete in $G_{R}$ and the quotient $G_{R} / \Gamma_{R}^{0}$ is compact.

Moreover, for the sake of simplicity, we assume throughout $\S 1$ that:

( $\Gamma 3) \Gamma$ is torsion-free; i.e., $\Gamma$ has no elements of finite order other than the identity element $I$.

It can be shown that a subgroup $\Gamma$ of $G$ satisfying $(\Gamma 1)$ and $(\Gamma 2)$ contains a subgroup with finite index which is torsion-free (cf. [1]).

$\S 1-2$. Now, the group $G_{R}=\mathrm{PSL}_{2}(R)$ acts on the complex upper half plane $\mathfrak{h}=\{z \in C \mid \operatorname{Im} z>0\}$ as :

$$
G_{R} \ni g_{R}= \pm\left(\begin{array}{ll}
a & b \\
c & d
\end{array}\right): \quad \mathfrak{h} \ni z \longrightarrow g_{R} \cdot z=\begin{aligned}
& a z+b \\
& c z+d
\end{aligned} \in \mathfrak{h} .
$$

For each $z \in \mathfrak{h}$, put

$$
\Gamma_{z}=\left\{\gamma \in \Gamma \mid \gamma_{R} \cdot z=z\right\} .
$$

Proposition. If $z \in \mathfrak{h}$ is such that $\Gamma_{z} \neq\{I\}$, then $\Gamma_{z}$ is an infinite cyclic group. Moreover, if $\gamma \in \Gamma_{z}, \gamma \neq I$, and if $\pm\left\{\lambda_{p}, \lambda_{,}^{-1}\right\}$ are the eigenvalues of $\gamma_{p} \in G_{p}=\operatorname{PSL}_{2}\left(k_{p}\right)$, then $\lambda_{p}, \lambda_{j}^{-1}$ belong to $k_{p}$, and do not belong to the group of $\mathfrak{p}$-adic units $\mathrm{n}_{\mathrm{p}}^{\times}$. In particular, they are distinct.

In fact, put $G_{z, R}=\left\{g_{R} \in G_{R} \mid g_{R} \cdot z=z\right\}$. Then, $G_{z, R}$ is isomorphic to the one-dimensional real torus group $T$, and hence is abelian. Now we have $\left(\Gamma_{z}\right)_{R} \cong \Gamma_{z} \cong\left(\Gamma_{z}\right)_{p}$ canonically, and since $\Gamma_{z} \neq\{I\}$ and $\Gamma_{z}$ is torsion-free, it is infinite. So, $\left(\Gamma_{z}\right)_{R}$ is an infinite subgroup of $G_{z, R} \cong T$, and hence is abelian (and dense in $G_{z, R}$ ). But $\Gamma_{z}$, as a subgroup of $\Gamma$, must be discrete in $G$, and so (since $\left(\Gamma_{z}\right)_{R}$ is dense in the compact subgroup $G_{z, R}$ of $\left.G_{R}\right),\left(\Gamma_{z}\right)_{\mathfrak{p}}$ must be discrete in $G_{p}$. Therefore, $\left(\Gamma_{z}\right)_{\mathfrak{p}}$ is a torsion-free, discrete abelian subgroup of $G_{p}$. Now our proposition follows easily from this. q. e.d.

We shall call an element $\gamma(\neq I)$ of $\Gamma$ elliptic if it is contained in $\Gamma_{z}$ for some $z \in \mathfrak{h}$. So, $\gamma \in \Gamma$ is elliptic if and only if $\gamma_{R}$ has imaginary eigenvalues, and for such $\gamma$, the point $z \in \mathfrak{h}$ such that $\gamma \in \Gamma_{z}$ is unique. So, we may denote such $\gamma$ also by $\gamma=\gamma_{z}$. Since the ellipticity remains unchanged by taking $\Gamma$ conjugate elements, we shall call the $\Gamma$-conjugacy class $\{\gamma\}_{\Gamma}$ containing $\gamma$ elliptic if $\gamma$ is so. For any elliptic $\Gamma$-conjugacy class $\{\gamma\}_{\Gamma}$, we shall define its degree, $\operatorname{deg}\{\gamma\}_{\Gamma}$, by 


$$
\operatorname{deg}\{\gamma\}_{\Gamma}=\left|V_{p}\left(\lambda_{p}\right)\right|,
$$

where $\pm\left\{\lambda_{p}, \lambda_{p}^{-1}\right\}$ are the eigenvalues of $\gamma_{\mathfrak{p}}$, and $V_{\mathfrak{p}}$ is the normalized additive valuation of $k_{p}$. Since $\lambda_{p} \notin \mathfrak{p}_{p}^{\times}$, $\operatorname{deg}\{\gamma\}_{\Gamma}$ is a positive integer, and it is clear that for any integer $r$, we have $\operatorname{deg}\left\{\gamma^{r}\right\}_{\Gamma}=|\gamma| \operatorname{deg}\{\gamma\}_{\Gamma}$. The following lemma makes clear the relation between $\operatorname{deg}\{\gamma\}_{\Gamma}$ and the elementary divisors of $\gamma_{p}$. It is well-known in elementary divisor theory that for any $x_{p} \in G_{p}$, there exist $u_{\mathfrak{p}}, u_{p}^{\prime} \in U_{\mathfrak{p}}=\mathrm{PSL}_{2}\left(\mathrm{D}_{\mathfrak{p}}\right)$ such that $u_{\mathfrak{p}} x_{\mathfrak{p}} u_{\mathfrak{p}}^{\prime}$ is of the form $\pm\left(\begin{array}{cc}\alpha_{\mathfrak{p}} & 0 \\ 0 & \alpha_{\mathfrak{p}}^{-1}\end{array}\right)$ with some $\alpha_{\mathfrak{p}} \in k_{\mathfrak{p}}$, and that $l\left(x_{p}\right)=\left|V_{\mathfrak{p}}\left(\alpha_{p}\right)\right|$ depends only on $x_{\mathfrak{p}}$, and not on the special choice of $u_{p}, u_{p}^{\prime} \in U_{p}$.

Lemma. Let $\{\gamma\}_{\Gamma}$ be an elliptic $\Gamma$-conjugacy class. Then, we have

$$
\operatorname{deg}\{\gamma\}_{\Gamma}=\operatorname{Min}_{\delta \in \Gamma} l\left(\left(\delta^{-1} \gamma \delta\right)_{p}\right)=\operatorname{Min}_{x_{p} \in G_{p}} l\left(x_{p}^{-1} \gamma_{p} x_{p}\right) .
$$

$\S 1$-3. Consider all points $z \in \mathfrak{h}$ such that $\Gamma_{z} \neq\{I\}$. Such points will be called $\Gamma$-fixed points. Two $\Gamma$-fixed points $z$, $z^{\prime}$ will be called $\Gamma$-equivalent if there exists $\gamma \in \Gamma$ such that $z^{\prime}=\gamma_{R} \cdot z$. We shall denote by $\mathfrak{S}(\Gamma)$ the totality of all $\Gamma$-equivalence classes of all $\Gamma$-fixed points. Then, it is clear that $\mathfrak{B}(\Gamma)$ can be identified with the totality of all $\Gamma$-conjugacy classes of all subgroups $\Gamma_{z}$, where $z$ runs over all $\Gamma$-fixed points. We shall call an elliptic element $\gamma=\gamma_{z}$ primitive if it generates $\Gamma_{z}$. Being primitive or not also remains unchanged by taking $\Gamma$-conjugate elements, and so we shall call $\{\gamma\}_{\Gamma}$ primitive if $\gamma$ is so.

Proposition. Let $\{\gamma\}_{\Gamma}$ be an elliptic $\Gamma$-conjugacy class. Then, $\left\{\gamma^{-1}\right\}_{\Gamma} \neq\{\gamma\}_{\Gamma}$.

In fact, it is enough to show that $\gamma_{R}^{-1}$ and $\gamma_{R}$ are not conjugate in $G_{R}=$ $\operatorname{PSL}_{2}(R)$. Suppose, on the contrary, that we had $\gamma_{R}^{-1}=g_{R}^{-1} \gamma_{R} g_{R}$ with $g_{R} \in G_{R}$. Then, if $z$ is the fixed point of $\gamma_{R}, g_{R}^{-1} z$ is also fixed by $\gamma_{R}$; hence $g_{R}^{-1} z=z$, and hence $g_{R}^{-1}$ commutes with $\gamma_{R}$. So, we get $\gamma_{R}^{-1}=\gamma_{R}$, which is impossible since $\Gamma$ is torsion-free. q.e.d.

So, there is a natural one-to-two correspondence between $\mathfrak{F}(\Gamma)$ and the set of all primitive elliptic $\Gamma$-conjugacy classes:

$$
\mathfrak{P}(\Gamma) \ni\left\{\Gamma_{z}\right\}_{\Gamma} \longleftrightarrow\left\{\gamma_{z}\right\}_{\Gamma},\left\{\gamma_{z}^{-1}\right\}_{\Gamma} .
$$

Finally, for each $P \in \mathfrak{F}(\Gamma)$, we put

$$
\operatorname{deg} P=\operatorname{deg}\{\gamma\}_{\Gamma}=\operatorname{deg}\left\{\gamma^{-1}\right\}_{\Gamma},
$$

where $\left\{\gamma^{ \pm 1}\right\}_{\Gamma}$ are the primitive elliptic $\Gamma$-conjugacy classes which correspond to $P$.

$\S 1-4$. Now we shall define the $\zeta$ function $\zeta_{\Gamma}(u)$ of $\Gamma$ by

$$
\zeta_{\Gamma}(u)=\prod_{P \in \mathfrak{P}(\Gamma)}\left(1-u^{\operatorname{deg} P}\right)^{-1},
$$


or, equivalently, by:

with

$$
\left\{\begin{array}{l}
\zeta_{\Gamma^{(}}(0)=1 \\
\log \zeta_{\Gamma^{(}}(u)=\sum_{m=1}^{\infty} N_{m} u^{m} / m
\end{array}\right.
$$

$$
N_{m}=\sum_{\substack{P \in \Re(T) \\ \operatorname{deg} P \mid m}} \operatorname{deg} P \quad(m \geqq 1) .
$$

Then, we can prove that $N_{m}$ are finite, and after some lengthy computation, we get the following result.

THEOREM 1. Our $\zeta$ function $\zeta_{\Gamma}(u)$ is a rational function of $u$ of the form:

$$
\zeta_{\Gamma}(u)=\frac{Q(u)}{(1-u)\left(1-q^{2} u\right)} \times(1-u)^{(q-1)(g-1)},
$$

where $q=N \mathfrak{p}, g$ is the genus of the Riemann surface $\mathfrak{h} / \Gamma_{R}^{0}$, and $Q(u)$ is a polynomial of $u$ of degree $2 g$ with rational integral coefficients and with $Q(0)=1$, satisfying the functional equation

$$
(q u)^{2 g} Q\left(q^{-2} u^{-1}\right)=Q(u) ;
$$

or equivalently, $Q(u)$ is of the form:

$$
Q(u)=\prod_{i=1}^{g}\left(1-\alpha_{i} u\right)\left(1-\alpha_{i}^{\prime} u\right), \quad \text { with } \quad \alpha_{i} \alpha_{i}^{\prime}=q^{2}(1 \leqq i \leqq g) .
$$

We also have an inequality:

$$
\left|\alpha_{i}\right|,\left|\alpha_{i}^{\prime}\right| \leqq q^{2}, \quad \alpha_{i}, \alpha_{i}^{\prime} \neq 1, q^{2} \quad(1 \leqq i \leqq g) .
$$

As for the proof, and for more explicit determination of $Q(u)$, cf. [1].

REMARK. If we do not assume $(\Gamma 3)$, then we must modify the definitions: of $\mathfrak{F}(\Gamma), \zeta_{\Gamma}(u)$, etc. But under a suitable modification, our Theorem 1 holds also for such general case.

§1-5. Some properties of $\Gamma$. Here, we shall state, without proof, some simple properties of the group $\Gamma$ satisfying $(\Gamma 1)$ and $(\Gamma 2)$. As for the proof, cf. [1].

(i). $\quad \Gamma$ is finitely generated and defined by a finite number of relations.

(ii). $\Gamma$ is residually finite, i. e., the intersection of all subgroups of $\Gamma$ with: finite indices is $\{I\}$.

(iii). $\Gamma$ contains a subgroup $\Gamma^{\prime}$ with finite index which is torsion-free.

(iv). The commutator subgroup $[\Gamma, \Gamma]$ of $\Gamma$ is of finite index in $\Gamma$, and if $\Gamma$ is torsion-free, then the group index $(\Gamma:[\Gamma, \Gamma])$ is a divisor of $Q(1)^{2}$, where $Q(u)$ is the numerator of the main factor of $\zeta_{\Gamma}(u)$ (see Theorem 1).

(v). $\Gamma$ has no non-trivial deformation in $G$. 


\section{$\S 2$. Analytic theory of $\mathrm{PSL}_{2}\left(k_{p}\right)$-fields}

Throughout $\S 2, \Gamma$ is a subgroup of $G=G_{R} \times G_{\mathfrak{p}}$ satisfying $(\Gamma 1)$ and $(\Gamma 2)$, and $Q$ resp. $C$ are the fields of rational numbers resp. complex numbers.

§ 2-1. As before, let $U_{\mathfrak{p}}=\operatorname{PSL}_{2}\left(\mathfrak{D}_{\mathfrak{p}}\right)$, and put

$$
U_{p}^{0}=U_{p}, \quad U_{p}^{n}=\left\{x \in \mathrm{SL}_{2}\left(\mathfrak{D}_{\mathfrak{p}}\right) \mid x \equiv \pm 1\left(\bmod \mathfrak{p}^{n}\right)\right\} / \pm 1 \quad(n \geqq 1) .
$$

So we have a descending sequence $U_{p}^{0} \supset U_{\uparrow}^{1} \supset \cdots$ of open compact subgroups of $G_{p}$ satisfying $\bigcap_{n=0}^{\infty} U_{p}^{n}=\{I\}$. Put

$$
\Gamma^{n}=\Gamma \cap\left(G_{R} \times U_{\uparrow}^{n}\right) \quad(n \geqq 0) .
$$

Remark that $\Gamma^{0}$ agrees with the previously defined one. So, we have a descending sequence $\Gamma_{R}^{0} \supset \Gamma_{R}^{1} \supset \cdots$ of discrete subgroups of $G_{R}$ with compact quotients, where each $\Gamma_{R}^{n}=\left(\Gamma^{n}\right)_{R}$ is a normal subgroup of $\Gamma_{R}^{0}$ with finite index, and $\bigcap_{n=0}^{\infty} \Gamma_{R}^{n}=\{I\}$. Now, let $L_{n}(n \geqq 0)$ be the field of automorphic functions with respect to $\Gamma_{R}^{n}$, i. e., $L_{n}$ is the field of all meromorphic functions $f(z)$ on the complex upper half plane $\mathfrak{h}$ which are invariant by the action of $\Gamma_{R}^{n}$ on $\mathfrak{h}$. So, we have an increasing sequence $L_{0} \subset L_{1} \subset \cdots$ of algebraic function fields of one variable over the complex number field $C$, where each $L_{n}$ is a finite Galois extension of $L_{0}$. We remark that we can take $n_{0} \geqq 0$ such that $U_{\mathfrak{p}}^{n_{0}}$ (and hence a priori $\Gamma_{R}^{n_{0}}$ ) is torsion-free. So, for such $n_{0}$ and for any $n \geqq n_{0}$, the extension $L_{n} / L_{n_{0}}$ is unramified.

Now, put $L=\bigcup_{n=0}^{\infty} L_{n}$. Then $G_{p}$ acts effectively on $L$ as a group of automorphisms* of $L$ over $C$ in the following manner. Let $g_{\mathfrak{p}} \in G_{p}$ and $f(z) \in L$. Take any $n \geqq 0$ such that $f(z) \in L_{n}$, and take any $\gamma \in \Gamma \cap\left(G_{R} \times U_{p}^{n} g_{p}^{-1}\right)(\neq \phi$, since $\Gamma_{\mathfrak{p}}$ is dense in $G_{\mathfrak{p}}$ by $\left.(\Gamma 1)\right)$. Then, $f\left(\gamma_{R} \cdot z\right)$ depends only on $f(z)$ and $g_{\mathfrak{p}}$, and does not depend on the choices of $n$ and $\gamma$. So, put $g_{p}(f(z))=f\left(\gamma_{R} \cdot z\right)$. Then, it can be checked immediately that for given $g_{\mathfrak{p}}$, the map $L \ni f(z) \rightarrow$ $g_{p}(f(z)) \in L$ is an automorphism of $L$ over $C$, that $g_{p}\left(h_{p}(f(z))\right)=\left(g_{p} h_{p}\right) f(z)$ holds for any $g_{\mathfrak{p}}, h_{\mathfrak{p}} \in G_{\mathfrak{p}}$ and $f(z) \in L$; and that this action of $G_{\mathfrak{p}}$ on $L$ is effective. Moreover, it is clear that for each $n \geqq 0, U_{p}^{n}$ acts trivially on $L_{n}$, that $U_{\mathfrak{p}}^{n}$ is the group of all automorphisms of $L$ over $L_{n}$, and that the only $G_{p}$-fixed elements of $L$ are elements of $C$.

In general, let $k$ be a field, and let $L$ be the union of an increasing sequence $L_{0} \subset L_{1} \subset \cdots \subset L_{n} \subset \cdots$ of algebraic function fields of one variable** with the

* By this, we do not mean that $G_{\mathfrak{p}}$ is the group of all automorphisms of $L$ over $C$.

** By this, we mean that each $L_{n}$ is a regular extension of $k$ (i.e., $L_{n}$ is finitely andi separably generated over $k$, and $k$ is algebraically closed in $L_{n}$ ), and $\operatorname{dim}_{k} L_{n}=1$. 
common constant field $k$, satisfying the following.

(L1) There exists $n_{0} \geqq 0$ such that for any $n \geqq n_{\theta}, L_{n}$ is unramified over $L_{n_{0}}$.

(L2) The group $G_{p}=\mathrm{PSL}_{2}\left(k_{p}\right)$ acts effectively on $L$ as a group of automorphisms of $L$ over $k$ in such a way that the Galois theory holds between $L_{n}$ and $U_{p}^{n}$ for each $n \geqq 0$; namely, $U_{p}^{n}$ acts trivially on $L_{n}$, and $U_{p}^{n}$ is the group of all automorphisms of $L$ over $L_{n}$. Moreover, we assume that the only $G_{p}$-fixed elements of $L$ are elements of $k$.

Here too, we do not mean that $G_{p}$ is the group of all automorphisms of $L$ over $k$.

Now, such a pair $\left\{L / k, G_{\mathfrak{p}}\right\}$ satisfying $(L 1)$ and $(L 2)$ will be called simply $a G_{p}$-field over $k$. Two such $\left\{L / k, G_{p}\right\}$ and $\left\{L^{\prime} / k, G_{\mathfrak{p}}\right\}$, with the common $k$ and $G_{p}$, are called isomorphic if there exists an isomorphism of $L$ onto $L^{\prime}$ over $k$ that commutes with the action of $G_{p}$.

We have shown that if $\Gamma$ is given, then we can construct $\left\{L / C, G_{p}\right\}$, a $G_{p}$-field over the complex number field $C$. Conversely, we can show that given any $\left\{L / C, G_{\mathfrak{p}}\right\}$, a subgroup $\Gamma$ of $G=G_{R} \times G_{\mathfrak{p}}$ satisfying $(\Gamma 1)$ and $(\Gamma 2)$ is defined; and that in this manner subgroups $\Gamma$ of $G$ satisfying $(\Gamma 1)$ and $(\Gamma 2)$ are in one-to-one correspondence with all $\left\{L / C, G_{\mathfrak{p}}\right\}$, where $\Gamma$ are considered up to conjugacy in $G$, and $\left\{L / C, G_{p}\right\}$, up to isomorphisms. Let us sketch the proof. Given $\left\{L / C, G_{\mathfrak{p}}\right\}$, consider the set $\Sigma$ of all non-equivalent places of $L$ over $C$. Then $\Sigma$ carries a natural complex structure, by which $\Sigma$ is a one dimensional complex manifold, each connected component of which is isomorphic to the complex upper half plane* $\mathfrak{h}$. The group $G_{\mathfrak{p}}$ acts effectively on $\Sigma$ as a group of automorphisms in a natural manner, and hence acts as a permutation group on the set of all connected components of $\Sigma$. Moreover, for each $n \geqq 0, U_{\Downarrow}^{n}$ acts transitively on the set of all connected components of $\Sigma$, and hence if we take any connected component $\Sigma_{0}$ of $\Sigma$ and put

$$
\Gamma_{\mathfrak{p}}=\left\{g_{\mathfrak{p}} \in G_{\mathfrak{p}} \mid g_{\mathfrak{p}} \cdot \Sigma_{0}=\Sigma_{0}\right\}^{* *},
$$

then $\Gamma_{\mathfrak{p}}$ is a dense subgroup of $G_{\mathfrak{p}}$. On the other hand, $\Gamma_{\mathfrak{p}}$ acts on $\Sigma_{0} \cong \mathfrak{h}$ as a group of automorphisms, and hence can be identified with a subgroup $\Gamma_{k}$ of $G_{R}=\mathrm{PSL}_{2}(R) \cong$ the automorphism group of $\mathfrak{h}$. Let $\Gamma$ be the subgroup of $G=$ $G_{R} \times G_{p}$ formed of all $\gamma_{R} \times \gamma_{p}$, where $\gamma_{R}, \gamma_{p}$ are corresponding elements of $\Gamma_{R}$, $\Gamma_{\mathrm{p}}$ respectively. Then, we can show that $\Gamma$ satisfies $(\Gamma 1)$ and $(\Gamma 2)$, and that this process of obtaining $\Gamma$ from $\left\{L / C, G_{p}\right\}$ is the inverse of the process of construction of $\left\{L / C, G_{p}\right\}$ from $\Gamma$ described at the beginning of $\S 2$. We sum-

* It is easy to see that each connected component of $\Sigma$ is isomorphic to $\mathrm{H} / \Delta$, with some discrete subgroup $\Delta$ of $G_{R}$; but it is less trivial to show that $\Delta=\{I\}$.

** So, there are as many connected components of $\Sigma$ as $G_{p} / I_{p}$. Its cardinal number is $\boldsymbol{\aleph}$-infinity. 
marize it in the following theorem.

THEOREM 2. Subgroups $\Gamma$ of $G=G_{R} \times G_{\mathfrak{p}}$ satisfying $(\Gamma 1)$ and $(\Gamma 2)$ are in one-to-one correspondence with $G_{p}$-fields $L$ over $C$.

§2-2. Now let us fix $\Gamma$ and the corresponding $\left\{L / C, G_{p}\right\}$. Then, if $\Delta$ is a subgroup of $G$ containing $\Gamma$ as a subgroup of finite index, and if $\left\{M / C, G_{p}\right\}$ is the $G_{p}$-field which corresponds to $\Delta$, then we can regard $M$ as a $G_{p}$-invariant subfield of $L$ containing $C$, where the action of $G_{p}$ on $M$ coincides with the restriction to $M$ of the action of $G_{p}$ on $L$. We also have $[L: M]=(\Delta: \Gamma)<\infty$. Conversely, if $M$ is any $G_{p}$-invariant subfield of $L$ such that $M \supsetneq C$, then we can show easily that $[L: M]<\infty$ and that $\left\{M / C, G_{p}\right\}$ is a $G_{p}$-field obtained from some $\Delta \supset \Gamma$ with $(\Delta: \Gamma)=[L: M]<\infty$. So, we have :

Proposition. Given $\Gamma$ and the corresponding $\left\{L / C, G_{p}\right\}$, subgroups $\Delta \supset \Gamma$ of $G$ with $(\Delta: \Gamma)<\infty$ and $G_{\mathfrak{p}}$-invariant subfields $M$ of $L$ with $M \nsupseteq C$ correspond in a one-to-one manner.

We shall call $\Gamma$ maximal if there is no such $\Delta$ other than $\Gamma$ itself, and $\left\{L / C, G_{p}\right\}$ irreducible if there is no such $M$ other than $L$ itself. So if $\Gamma$ corresponds to $\left\{L / C, G_{p}\right\}$, then,

$$
\left\{L / C, G_{p}\right\} \text { is irreducible if and only if } \Gamma \text { is maximal. }
$$

We remark that any $\Gamma$ is contained in a maximal one*. In fact, if $\Delta \supset \Gamma$ and $(\Delta: \Gamma)<\infty$, then, since $\Gamma_{\mathfrak{p}}$ is dense in $G_{\mathfrak{p}}$ we have $\Delta_{\mathfrak{p}}=\Delta_{\mathfrak{p}}^{n} \cdot \Gamma_{\mathfrak{p}}$ for any $n \geqq 0$, and hence $(\Delta: \Gamma)=\left(\Delta_{R}^{n}: \Gamma_{R}^{n}\right)$ for any $n \geqq 0$. But $\Gamma_{R}^{0}$ is a discrete subgroup of $G_{R}$ with compact quotient, and hence $\left(\Delta_{R}^{0}: \Gamma_{R}^{0}\right)$, and hence also $(\Delta: \Gamma)$, is bounded. This also shows that if $\Gamma_{R}^{0}$ is a maximal Fuchsian group, then $\Gamma$ itself is maximal.

$\S 2$-3. Let $\left\{L / C, G_{p}\right\}$ be any $G_{p}$-field over $C$, and let Aut $(L / C)$ be the group of all automorphisms of the field $L$ which are trivial on $C$. Then, by the definition of $\left\{L / C, G_{p}\right\}$, the group $G_{p}$ is contained in Aut $(L / C)$, but may not coincide with the whole group Aut $(L / C)$. The following theorem is basic for our later studies (as for the proof, cf. [1]).

THEOREM 3. The group $G_{\mathfrak{p}}$ is a characteristic subgroup of Aut $(L / C)$ with finite index.

Here, the essential point is the finiteness of the group index (Aut $(L / C): G_{p}$ ); that $G_{\mathfrak{p}}$ is a characteristic subgroup follows immediately from this and from the simplicity of the group $G_{\mathfrak{p}}=\operatorname{PSL}_{2}\left(k_{\mathfrak{p}}\right)$.

COROLlary. The centralizer $Z$ of $G_{\mathfrak{p}}$ in $\operatorname{Aut}(L / C)$ is finite. If, moreover, $\left\{L / C, G_{p}\right\}$ is irreducible, then $Z=\{I\}$.

* Actually, we can prove moreover that for given $\Gamma$, there are at most finitely many $\Delta$. So, for given $\left\{L / C, G_{\mathfrak{p}}\right\}$, the number of $M$ is also finite. 
In fact, $Z \cap G_{p}$ is contained in the center of $G_{p}$, and hence is trivial; and since (Aut $\left.(L / C): G_{p}\right)<\infty, Z$ is finite. So, let $M$ be the subfield of $L$ corresponding to the finite automorphism group $Z$. Then $M$ is $G_{p}$-invariant, and obviously $M \supsetneq C$. So, if $\left\{L / C, G_{p}\right\}$ is irreducible, we get $M=L$, and hence $Z=\{I\}$.

$\S 2-4$. Let $\left\{L / C, G_{p}\right\}$ be a $G_{p}$-field over $C$. A subfield $L_{k}$ of $L$ is called $a$ good subfield of $\left\{L / C, G_{p}\right\}$ over $k=L_{k} \cap C$ if $L_{k}$ is $G_{p}$-invariant and if $L_{k} \cdot C$ $=L$. In this situation, it follows easily that $L_{k}$ and $C$ must be linearly disjoint over $k$. If $L_{k}$ is a good subfield of $\left\{L / C, G_{p}\right\}$ over $k$, then it follows easily that $\left\{L_{k} / k, G_{p}\right\}$ is also a $G_{p}$-field over $k$; and for any $k^{\prime}$ with $k \subset k^{\prime} \subset C$, the composite $L_{k^{\prime}}=L_{k} \cdot k^{\prime}$ is a good subfield of $\left\{L / C, G_{\mathfrak{p}}\right\}$ over $k^{\prime}$. Now our main theorem of $\S 2$ runs as follows.

Theorem 4. Let $\left\{L / C, G_{p}\right\}$ be a Gp-field over $C$. Then, there exists an algebraic number field $k$ of finite degree over $Q$ and a good subfield $L_{k}$ of $\left\{L / C, G_{\mathfrak{p}}\right\}$ over $k$. If, moreover, the centralizer $Z$ of $G_{p}$ in $\operatorname{Aut}(L / C)$ is trivial, then good subfields of $\left\{L / C, G_{p}\right\}$ are essentially unique; namely, there exists a good subfield $L_{k_{0}}$ over an algebraic number field $k_{0}$ of finite degree, such that all other good subfields are those obtained by the composite $L_{k_{0}} \cdot k$ of $L_{k_{0}}$ and fields $k$ with $k_{0} \subset k \subset C$. (On the other hand, if $Z \neq\{I\}$, then good subfields are not essentially unique.)

As for the proof, cf. [1]. Unfortunately, it is too lengthy to be reproduced here. It is based on property (v) of $\S 1-5$, Theorems 2 and 3 , and on some group theoretical properties of $G_{p}$.

By this, and by the corollary of Theorem 3, we get:

COROLlary 1. If $\left\{L / C, G_{p}\right\}$ is irreducible, then it has an essentially unique good subfield $L_{k_{0}}$ over an algebraic number field $k_{0}$ of finite degree.

In short, any $G_{p}$-field over the complex number field $C$ is the constant field extension of some $G_{p}$-field over an algebraic number field; and if the former is irreducible, then it is (essentially) uniquely so. It is a fundamental open problem to determine the relation between this $k_{0}$ and $k_{p}$.

So far, we defined good subfields $L_{k}$ over $k$ to be $G_{p}$-invariant subfields of $L$ satisfying $L_{k} \cdot C=L, L_{k} \cap C=k$, and looked for "smallest possible" good subfields. However, we can impose stronger conditions on the definition of good subfields ; for example, instead of $G_{p}$-invariance, we can impose Aut $(L / C)$ invariance. With this definition, Theorem 4 is also valid, and the condition of the triviality of the centralizer of $G_{\triangleright}$ in $A u t(L / C)$ can be replaced by the triviality of the center of Aut $(L / C)$; and thus we get the essential uniqueness of good subfields for a wider class of $\left\{L / C, G_{p}\right\}$.

Finally, let $\bar{Q}$ be the algebraic closure of the field of rational numbers $Q$, and let $k^{\prime}$ be any field with $\bar{Q} \subset k^{\prime} \subset C$. Let $\left\{L / C, G_{p}\right\}$ be any $G_{p}$-field over $C$. 
So, $L$ can be considered as defined from $\Gamma$ as in $\S 2-1$. Let the definition of good subfields be either of the above defined ones. Then, by Theorem 1, there is a good subfield $L_{k}$ over an algebraic number field $k$, and hence there is a good subfield $L_{k^{\prime}}=L_{k} \cdot k^{\prime}$ over $k^{\prime}$. On the other hand, we can show easily that if $L_{k^{\prime}}$ is any good subfield of $\left\{L / C, G_{p}\right\}$ over $k^{\prime}$ with $\bar{Q} \subset k^{\prime} \subset C$, then $L_{k^{\prime}}$ consists of all $f(z) \in L$ such that the values of $f$ at all $\Gamma$-fixed points are contained in $k^{\prime} \cup\{\infty\}$. So, we get:

COROLlary 2. If $\bar{Q} \subset k^{\prime} \subset C$, then there is a unique good subfield $L_{k^{\prime}}$ of $\left\{L / C, G_{p}\right\}$ over $k^{\prime}$. It consists of all $f(z) \in L$ whose values at all $\Gamma$-fixed points are contained in $k^{\prime} \cup\{\infty\}$. (So, of course, $L_{k^{\prime}}=L_{\bar{Q}} \cdot k^{\prime}$.)

\section{$\S 3$. Conjectures (The congruence monodromy problems)}

$\S 3-1$. Now, we are in the situation to state our series of conjectures explicitly.

Conjecture 0. With each $\Gamma$ satisfying $(\Gamma 1),(\Gamma 2)$ and $(\Gamma 3)$, we can associate an algebraic function field $K$ of one variable with finite constant field $F_{q^{2}}$ and with genus $g$, satisfying the properties stated in the following conjectures. Here, $q=N \mathfrak{p}$, and $g$ is the genus* of $\mathfrak{h} / \Gamma_{R}^{0}$.

CONJECTURE 1. Denoting by $\mathfrak{B}(K)$ the set of all prime divisors of $K$ over $F_{q^{2}}$, there exists a finite subset $\subseteq(K)$ of $\mathfrak{B}(K)$ consisting of $(q-1)(g-1)$ prime divisors of $K$ of degree one over $F_{q^{2}}$, and a one-to-one correspondence between $\mathfrak{F}(\Gamma)$ and $\mathfrak{B}(K)-\mathfrak{S}(K)$ which preserves the degrees of elements of $\mathfrak{B}(\Gamma)$ and of $\mathfrak{B}(K)-\subseteq(K)$;

$$
\mathfrak{P}(K)-\widetilde{S}(K) \underset{1: 1}{\longleftrightarrow} \mathfrak{P}(\Gamma),
$$

and which agrees with the following conjectures.

CONJECTURE 2. There exists a one-to-one correspondence between the set of all subgroups $\Gamma^{\prime}$ of $\Gamma$ with finite indices and that of all finite extensions $K^{\prime}$ of $K$ satisfying the following conditions (i), (ii).

(i) $K^{\prime} / K$ is unramifild.

(ii) All prime divisors of $K$ belonging to $\subseteq(K)$ are decomposed completely in $K^{\prime}$.

Moreover, this one-to-one correspondence $K^{\prime} \leftrightarrow \Gamma^{\prime}$ satisfies the Galois theory.

REMARK. The constant field of $K^{\prime}$ must also be $F_{q^{2}}$, because of (ii).

Now let $\Re$ be the union of all $K^{\prime}$ satisfying (i), (ii). So, $\Re$ is the maximum unramified extension of $K$ in which all prime divisors $P$ of $K$ with $P \in \subseteq(K)$ are decomposed completely. Then, by the above conjecture 2, the Galois group

* Or, what is the same, the genus of the function field $L_{0}$. Since $\Gamma$, and hence $\Gamma_{R}^{0}$, is torsion-free, we have $g \geqq 2$. 
$\mathscr{B}=\mathbb{S}(\mathscr{R} / K)$ of $\mathscr{K}$ over $K$ can be identified with the projective limit of all finite factor groups of $\Gamma$. But we know that $\Gamma$ is residually finite (see $\S 1-5$, (ii)), and hence the natural homomorphism of $\Gamma$ into $\mathbb{S}$ is injective. So, we shall consider $\Gamma$ as a (dense) subgroup of $\mathbb{B}$. For each prime divisor $P \in \mathfrak{S}(K)-$ $\widetilde{S}(K)$, let $\left\{\gamma_{P}^{ \pm 1}\right\}_{\Gamma} \in \mathfrak{F}(\Gamma)$ be the corresponding mutually inverse pair of primitive elliptic $\Gamma$-conjugacy classes. Since $\Gamma$ is a subgroup of $\mathbb{S},\left\{\gamma_{P}^{ \pm 1}\right\}_{\Gamma}$ defines a mutually inverse pair of (\$)-conjugacy classes, which will be denoted by $\left\{\gamma_{P}^{ \pm 1}\right\}_{2}$. On the other hand, let $\left(\begin{array}{c}\Re / K \\ P\end{array}\right)$ be the Frobenius substitution of $P$ in $\mathfrak{R} / K$. It is a conjugacy class of the Galois group $\mathbb{B}$ of $\mathscr{R} / K$.

Conjecture $3^{*}$. The notations being as above, we have

$$
\left(\begin{array}{c}
\Re / K \\
P
\end{array}\right)=\left\{\gamma_{P}^{ \pm 1}\right\}_{3} \quad \text { for all } P \in \mathfrak{P}(K)-\widetilde{\varsigma}(K),
$$

where the sign \pm 1 is left as an ambiguity.

REMARK. If $P \in \subseteq(K)$, then, by conjecture 2 , we have $\left(\begin{array}{c}\Re / K \\ P\end{array}\right)=1$.

Finally, the notations being as above, $K^{\prime}$ is the field associated with $\Gamma^{\prime} ; \circlearrowleft\left(K^{\prime}\right)$ $i$ s the set of all prime divisors of $K^{\prime}$ which lie on $\subseteq(K)$; and by the notation of $\S 0-1,\left\{\gamma_{P}^{ \pm} f_{i}^{f}\right\}_{\Gamma^{\prime}}$ is the mutually inverse pair of primitive elliptic conjugacy classes of $\Gamma^{\prime}$ which corresponds to $P_{i}^{\prime}(1 \leqq i \leqq t$; where the suffices are assumed to be suitably chosen). So, $\mathscr{R}^{\prime}$ (i.e., $\Re$ for $K^{\prime}$ ) coincides with $\Re$.

$\S 3-2$. Connection with the results of $\S 1$. In $\S 1$, we defined the $\zeta$ function of $\Gamma ; \zeta_{\Gamma}(u)=\prod_{P \equiv \mathfrak{P}(\Gamma)}\left(1-u^{\operatorname{deg} P}\right)^{-1}$, and Theorem 1 asserts that it is of the form:

$$
\zeta_{\Gamma}(u)=\frac{\prod_{i=1}^{g}\left(1-\alpha_{i} u\right)\left(1-\alpha_{i}^{\prime} u\right)}{(1-u)\left(1-q^{2} u\right)} \times(1-u)^{(q-1)(g-1)} ; \quad \alpha_{i} \alpha_{i}^{\prime}=q^{2}(1 \leqq i \leqq g) .
$$

This suggests the possibility that the first factor of $\zeta_{\Gamma}(u)$, i. e.,

$$
\frac{\prod_{i=1}^{g}\left(1-\alpha_{i} u\right)\left(1-\alpha_{i}^{\prime} u\right)}{(1-u)\left(1-q^{2} u\right)}
$$

is a congruence $\zeta$ function of some algebraic function field $K$ of one variable with genus $g$ and with the constant field $F_{q^{2}}$. Now if it is so, then for each $n \geqq 1$, we have :

* This is a stronger form of the conjecture 3 given in the introduction. It does not contradict "Tschebotareff's density theorem". In fact, T's density theorem "on $\Gamma$. side" can be proved by using $Q\left(q^{-1}\right) \neq 0$ (Theorem 1$)$ and its generalizations to $L$-functions of $\Gamma$. 
the number of prime divisors of $K$ with degree $n$

$$
=\text { the number of } P \in \mathfrak{P}(\Gamma) \text { with degree } n+\left\{\begin{array}{l}
0 \cdots n>1 \\
(q-1)(g-1) \cdots n=1 .
\end{array}\right.
$$

Actually, this is not the original reason that motivated the author to entertain conjectures 0,1 ; but, in any case, this agrees with the conjectures 0,1 . As conjectures 0,1 were so, conjectures 2,3 were also suggested by the elliptic modular case (see $\S 4-2$, and [2]), and we cannot explain the reasonings of conjectures 2,3 only with the results of $\S 1$. The only thing we can say here is this; if we assume conjectures 2 and 3, then the reason for " the existence of $(q-1)(g-1)$ prime divisors of $K$ of degree one which do not correspond to any $\left\{\gamma_{P}^{ \pm 1}\right\}_{\Gamma} \in \mathfrak{F}(\Gamma)$ (conjecture 1 )" can be explained most clearly!

As for $\S 1-5$, we remark that it gives a pleasant exercise to interpret the assertions of $\S 1-5$ (ii), (iv) in view of our conjectures.

$\S 3-3$. Connection with the results of $\S 2$. This is more vague and incomplete. The motivation for the study of $\S 2$ lies in trying to obtain the questioned $K$ out of $\Gamma$. Our conjecture implicitly assumes that $K$ is obtained by "reduction mod $\Re$ " of $L_{0}$; and so, our study in $\S 2$ is the first thing that should be done along this line. Our results seem encouraging.

\section{$\S 4$. Examples}

§4-1. Let $F$ be a totally real algebraic number field of finite degree, and let $B$ be a divison quaternion algebra over $F$, in which all but one infinite prime of $F$ are ramified. Let $\mathfrak{p}$ be a finite prime of $F$ which is unramified in $B$, and let $\mathfrak{D}$ be an order of $B$ containing the maximal order of $F$. Put $\mathfrak{D}^{(\mathfrak{p})}=\bigcup_{n=0}^{\infty} \mathfrak{p}^{-n} \mathfrak{d}$, and let $\Gamma$ be the quotient by the center of the group of all elements $x$ of $B$ such that $x^{-\mathfrak{D}_{\mathfrak{D}}^{(p)}} x=\mathfrak{d}^{(\mathfrak{p})}$ and that $n(x) \in R^{2}, F_{\mathfrak{p}}^{2}$. Let $\iota_{\infty}$ resp. $\iota_{\mathfrak{p}}$ be the embeddings of $F$ into $R$ resp. $F_{\mathfrak{p}}$ defined with respect to the infinite prime of $F$ unramified in $B$ resp. p, where $F_{\mathfrak{p}}$ is the p-adic completion of $F$. Then the tensor products

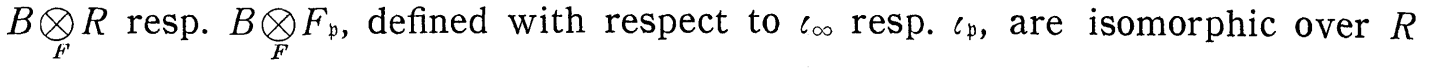
resp. $F_{\mathfrak{p}}$ to $M_{2}(R)$ resp. $M_{2}\left(F_{\mathfrak{p}}\right)$; and by this, we can regard $\Gamma$ as a subgroup of $G=\mathrm{PSL}_{2}(R) \times \mathrm{PSL}_{2}\left(F_{\mathfrak{p}}\right)$. Now, it is a simple exercise in arithmetic of algebraic groups to check that $\Gamma$ satisfies $(\Gamma 1)$ and $(\Gamma 2)$. On the other hand, up to commensurability, these are the only examples of $\Gamma$ satisfying $(\Gamma 1)$ and $(\Gamma 2)$ that we know so far. We do not know whether such $\Gamma$ satisfy "the congruence subgroup properties".

Now, for these $\Gamma$, the Fuchsian groups $\Gamma_{R}^{0}$ belong to those classes for which the quotients $\mathfrak{h} / \Gamma_{R}^{0}$ have been studied in detail by G. Shimura [3], where 
much of the deepest results on arithmetic of elliptic modular functions have been generalized to these cases by using his theory of moduli of abelian varieties. Although neither of our conjectures have been proved for these cases, it is expected that the study of these cases will help us give a further insight for the general case. The following is some information on these $\Gamma$ which we get directly from [3]. Here, we assume that $\mathfrak{D}$ is maximal.

(i) The $\zeta$ function. For such $\Gamma$, it follows directly from the results of [3] (and from our explicit formula for $Q(u), \mathrm{cf}$. [1] that the main factor

$$
\frac{Q(u)}{(1-u)\left(1-q^{2} u\right)}
$$

of $\zeta_{\Gamma}(u)$ coincides with the congruence $\zeta$ function of some algebraic function field $K$ over $F_{q^{2}}$, for almost all $\mathfrak{p}$. The field $K$ is obtained by "reduction $\bmod \mathfrak{F} "$ of $L_{0}$.

(ii) The field $k_{0}$ (in Theorem 4). Since $\mathfrak{o}$ is maximal, $\Gamma$ is maximal in the sense of $\S 2-2$, and hence we get an algebraic number field $k_{0}$. It turns out (by [3]) that the composite $k_{0} \cdot F$ is a class field over $F$, whose ideal class group can be explicitly written down. (Added in proof. $k_{0}$ contains $F$. Cf. [1].)

§4-2. Elliptic modular case ("quasi-example")*. Let $p$ be a rational prime number other than 2 or 3 , let $\boldsymbol{Z}$ be the ring of rational integers, and put

$$
\boldsymbol{Z}^{(p)}=\left\{a / p^{n} \mid a, n \in \boldsymbol{Z}\right\}, \quad \Gamma=\mathrm{PSL}_{2}\left(\boldsymbol{Z}^{(p)}\right) .
$$

Then $\Gamma$ can be considered as a discrete subgroup of $G=G_{R} \times G_{p}$, where $G_{R}=\operatorname{PSL}_{2}(R), G_{p}=\operatorname{PSL}_{2}\left(Q_{p}\right)$, and $Q_{p}$ is the $p$-adic number field. Let $Z_{p}$ be the ring of $p$-adic integers, put $U_{p}=\operatorname{PSL}_{2}\left(Z_{p}\right)$, and put $\Gamma^{0}=\Gamma \cap\left(G_{R} \times U_{p}\right)$. Then it is clear that $\Gamma^{0}=\operatorname{PSL}_{2}(Z)$, and hence its projection $\Gamma_{R}^{0}$ is a discrete subgroup of $G_{R}$, but the quotient is not compact. So, the quotient $G / \Gamma$ is not compact, but since $G_{R} / \Gamma_{R}^{0}$ has finite invariant volume, we see easily that $G / \Gamma$ also has finite invariant volume.

Now, since $G / \Gamma$ is not compact, we must modify our conjectures. The modified conjectures are partly proved for the above $\Gamma$ and its congruence subgroups, and the results are stated in [2]. As for the above $\Gamma=\operatorname{PSL}_{2}\left(\boldsymbol{Z}^{(m)}\right)$, conjecture 1 (modified) is true for $K=F_{p^{2}}(\widetilde{j})$, the rational function field, and here $\widetilde{S}(K)$ is the set of all supersingular moduli $j$. Namely, an element $j$ of a finite field of characteristic $p$ is called supersingular, if the elliptic curve with modulus $j$ has no points of order $p$; and it is known that such $j$ is contained in $F_{p^{2}}$ and hence defines a prime divisor of $K=F_{p^{2}}(\tilde{j})$ of degree one. Conjecture 2 (modified) is proved only for the pairs of such $\Gamma^{\prime}$ that contain

* As for the details of this section, cf. [2] for a rough sketch, and [1] for more detailed statements and proofs. 
some congruence subgroup of $\Gamma$ and such $K^{\prime}$ that are obtained by the division of the elliptic curve with modulus $\tilde{j}$. Whether they exhaust all $\Gamma^{\prime}$ and $K^{\prime}$ is an open problem. Conjecture 3 is true for these $\Gamma^{\prime}$ and $K^{\prime}$. All these are consequences of modern theory of elliptic curves (by M. Deuring, J. Igusa, etc.).

ADDED IN PROOF. After this paper was submitted, the author learned that J. Mennicke and J.P. Serre had succeeded in proving the congruence subgroup property for $\Gamma=\mathrm{PSL}_{2}\left(Z^{(p)}\right)$ (cf. [4]). Mennicke has stronger results on this group $\Gamma$, and Serre's results are on more general $\mathrm{SL}_{2}$ type groups. However, the first idea is due to Mennicke (cf. [4], [5]).

\section{University of Tokyo}

\section{References}

[1] Y. Ihara, On congruence monodromy problems, Lecture note at Princeton University, 1967 Spring Term, to appear.

[2] Y. Ihara, Algebraic curves modp and arithmetic groups, Proceedings of Symposia in Pure Mathematics, Vol. 9, 1966, p. 265-271.

[3] G. Shimura, Construction of class fields and zeta functions of algebraic curves, Ann. of Math., 85 (1967), 58-159.

[4] J. Mennicke, On Ihara's modular group, Invent. Math., 4 (1967), 202-228.

[5] J.P. Serre, Le problème des groupes de congruence pour $\mathrm{SL}_{2}$, (unpublished manuscript). 